\title{
Exact cosmological solution of the coupled Einstein-Majorana fermion-scalar field equations
}

\author{
A. B. Balantekin* \\ Department of Physics, University of Wisconsin, Madison, Wisconsin 53706 USA \\ T. Dereli ${ }^{\dagger}$ \\ Department of Physics, Koç University, 34450 Sariyer, Ístanbul, Turkey \\ (Received 25 May 2006; published 26 January 2007)
}

\begin{abstract}
We couple a neutral scalar field and a Majorana fermion field to Einstein gravity represented by the Robertson-Walker metric and find a class of exact cosmological solutions.

DOI: 10.1103/PhysRevD.75.024039

PACS numbers: 04.20.Jb, 14.60.Pq, 14.60.St, 98.80.Jk
\end{abstract}

\section{INTRODUCTION}

Recent years witnessed progress at a breathtaking pace both in cosmology and in neutrino physics. In cosmology, initial evidence for the acceleration of the universe from high red-shift supernova observations was subsequently verified by the precise measurements of the cosmic microwave background radiation. These observations and measurements discovered that there is a significant "darkenergy" component of the universe. In a parallel development solar, atmospheric, and reactor neutrino experiments firmly established that neutrinos are massive and that they mix. Mass-varying neutrinos were introduced in part as an attempt to explain the origin of the cosmological darkenergy density and why its magnitude is apparently coincidental with that of the measured neutrino mass splittings [1,2]. Such mass-varying neutrinos can behave as a negative pressure fluid leading to the acceleration of the universe.

Models for the dark energy in which the energy density of the scalar field approximates Einstein's cosmological constant were studied in detail [3]. It can be shown that in the mass-varying neutrino scenario dark energy is also equivalent to having a cosmological constant [4]. It has been argued that mass-varying neutrino models contain an instability when neutrinos become nonrelativistic and a stable neutrino-varying mass model is indistinguishable from a cosmological constant [5]. Mass-varying neutrino models without acceleron like scalar fields were also proposed [6]. However, an analysis of the cosmic microwave background radiation anisotropies and large scale structure implies some evidence for coupling between neutrinos and a scalar field [7].

Quantization of a Fermi field, coupled to the RobertsonWalker metric, had been worked out some time ago [8]. Motivated by the recent studies of mass-varying neutrinos, in this paper we explore if exact solutions exist when a neutral scalar field (representing the acceleron of the massvarying neutrino models) as well as a Majorana fermion

\footnotetext{
*Electronic address: baha@physics.wisc.edu

†Electronic address: tdereli@ku.edu.tr
}

field (representing the neutrino) couples to gravity via Robertson-Walker metric. We show that such solutions indeed exist.

In the next section we outline our model. In Sec. III we present our exact solution to this model. A brief discussion of our results in Sec. IV concludes the paper.

\section{THE COSMOLOGICAL MODEL}

We solve coupled Einstein-acceleron-Majorana neutrino field equations derived by a variational principle from the Lagrangian density

$$
\begin{aligned}
\mathcal{L}= & \frac{1}{2 \kappa} \mathcal{R} * 1-\frac{1}{2} d \phi \wedge * d \phi+V(\phi) * 1 \\
& +i \bar{\psi}(\gamma \wedge * \nabla) \psi-i M(\phi) \bar{\psi} \psi * 1
\end{aligned}
$$

where $\psi$ is the 4-component (Majorana spinor) sterile neutrino field and $\phi$ is the (real, scalar) acceleron field; $V(\phi)$ the acceleron potential function, $M(\phi)$ the varyingmass of the neutral fermion to be determined. $\kappa=8 \pi G$ is the gravitational coupling constant (in natural units such that $c=1=\hbar)$. The space-time geometry is given in terms of a metric tensor $g$ of Lorentzian signature -+++ and its unique Levi-Civita connection $\nabla \cdot \mathcal{R}$ is the corresponding curvature scalar and $* 1$ denotes the oriented volume element. We use a set of real $\gamma$-matrices (Majorana realization) $\left\{\gamma_{a}\right\}$ that satisfy

$$
\gamma_{a} \gamma_{b}+\gamma_{b} \gamma_{a}=2 \eta_{a b} I \text {. }
$$

Reality means $\gamma^{*}{ }_{a}=\gamma_{a}$. Hence we have $\gamma^{\dagger}{ }_{0}=\gamma^{T}{ }_{0}=$ $-\gamma_{0}$ and $\gamma_{i}^{\dagger}=\gamma_{i}^{T}=\gamma_{i}$. The conjugate spinor field is

$$
\bar{\psi}=\psi^{\dagger} C,
$$

where the charge conjugation matrix $C=\gamma_{0}$. With our conventions the spinor field $\psi$ is self-conjugate if and only if it is real. That is

$$
\psi^{C} \equiv C \bar{\psi}^{T}=\psi^{*} .
$$

We look for exact solutions of the coupled system of field equations in the Robertson-Walker space-time determined by the metric (spatially flat, $k=0$ ) 


$$
g=-d t \otimes d t+R^{2}(t)(d x \otimes d x+d y \otimes d y+d z \otimes d z)
$$

in terms of the cosmic time $t$ and isotropic coordinates $x^{i}:(x, y, z) . R(t) \geq 0$ is the expansion function. We further adopt the ansatz

$$
\phi=\phi(t)
$$

and

$$
\psi=\left(h_{1}(t)+h_{2}(t) \gamma_{0}\right) \xi, \quad \bar{\psi}=\bar{\xi}\left(h_{1}(t)-h_{2}(t) \gamma_{0}\right)
$$

where $h_{1}(t), h_{2}(t)$ are functions to be determined and $\xi$ is a constant Majorana 4-spinor. We substitute these in the variational field equations and reduce them to a set of ordinary differential equations:

$$
\begin{gathered}
\frac{3}{\kappa}\left(\frac{\dot{R}}{R}\right)^{2}-\frac{\dot{\phi}^{2}}{2}+V(\phi)=\mathcal{N} M(\phi)\left(h_{1}{ }^{2}+h_{2}^{2}\right) \\
\frac{2}{\kappa} \frac{\ddot{R}}{R}+\frac{1}{\kappa}\left(\frac{\dot{R}}{R}\right)^{2}+\frac{\dot{\phi}^{2}}{2}+V(\phi) \\
=\mathcal{N}\left(h_{2} \dot{h}_{1}-h_{1} \dot{h}_{2}\right)+\mathcal{N} M(\phi)\left(h_{1}^{2}+h_{2}^{2}\right) \\
\ddot{\phi}+3 \frac{\dot{R}}{R} \dot{\phi}-\frac{d V}{d \phi}=-\mathcal{N}\left(\frac{d M}{d \phi}\right)\left(h_{1}{ }^{2}+h_{2}{ }^{2}\right) \\
\dot{h}_{1}+\frac{3 \dot{R}}{2 R} h_{1}+M(\phi) h_{2}=0 \\
\dot{h}_{2}+\frac{3 \dot{R}}{2 R} h_{2}-M(\phi) h_{1}=0
\end{gathered}
$$

where

$$
i(\bar{\xi} \xi) \equiv \mathcal{N}
$$

may be (semiclassically) interpreted as a (real) vacuum expectation value.

Remark: 1 If we differentiate (8) and simplify by using (8) and (10)-(12) again, we obtain precisely (9). Thus, out of the five equations above, only four of them are independent. On the other hand we have four functions to solve for. Therefore the system is well-determined.

Remark: 2 If we use (11) and (12) for the derivatives of $h_{1}$ and $h_{2}$ and substitute in (9), the right-hand side vanishes. This means that in our simple model the neutrino pressure is zero.

Remark: 3 (11) and (12) implies that

$$
R^{3}\left(h_{1}^{2}+h_{2}^{2}\right)=C
$$

is a constant of motion. We designate $n=\mathcal{N} C$ which may be called the neutrino number density.

\section{AN EXACT SOLUTION}

We only consider the simple case of a constant potential. $V(\phi)=V_{0}$. First we solve the resulting equations,

$$
\begin{gathered}
\frac{2}{\kappa} \frac{\ddot{R}}{R}+\frac{1}{\kappa}\left(\frac{\dot{R}}{R}\right)^{2}+\frac{\dot{\phi}^{2}}{2}+V_{0}=0, \\
\frac{3}{\kappa}\left(\frac{\dot{R}}{R}\right)^{2}-\frac{\dot{\phi}^{2}}{2}+V_{0}-\frac{n}{R^{3}} M(\phi)=0, \\
\ddot{\phi}+3 \frac{\dot{R}}{R} \dot{\phi}+\frac{n}{R^{3}} \frac{d M}{d \phi}=0,
\end{gathered}
$$

by assuming the existence of solutions that satisfy

$$
R(t)=R_{0} e^{\alpha \phi(t)}
$$

where both $R_{0}$ and $\alpha$ are constants that may be chosen later. We then solve (15) and get

$$
\dot{\phi}(t)=\sqrt{\frac{2 \kappa V_{0}}{\kappa+6 \alpha^{2}}} \cot \left(\sqrt{\frac{\kappa V_{0}}{8 \alpha^{2}}\left(\kappa+6 \alpha^{2}\right)}\right) .
$$

Inserting this expression into (16) determines the functional form of $M(\phi)$. In order to get a closed expression for it, we first write $\phi$ as a function of $t$ :

$$
\phi(t)=\phi_{0}-\frac{2 \alpha}{\kappa+6 \alpha^{2}} \ln \left|\csc ^{2}\left(\sqrt{\frac{\kappa V_{0}}{8 \alpha^{2}}\left(\kappa+6 \alpha^{2}\right) t}\right)\right| .
$$

Here $\phi_{0}$ is an integration constant. Taking the exponential of both sides and using trigonometric identities we find

$$
\cot ^{2}\left(\sqrt{\frac{\kappa V_{0}}{8 \alpha^{2}}\left(\kappa+6 \alpha^{2}\right)} t\right)=e^{-\left(\kappa+6 \alpha^{2}\right) /(2 \alpha)\left(\phi-\phi_{0}\right)}-1 .
$$

Substituting this in (16), we solve for the mass function as a function of $\phi$ :

$$
\begin{aligned}
n M(\phi)= & \frac{2 \kappa V_{0} R_{0}^{3}}{\kappa+6 \alpha^{2}}\left\{e^{3 \alpha \phi}+\frac{6 \alpha^{2}-\kappa}{2 \kappa} e^{\left(\left(\kappa+6 \alpha^{2}\right) /(2 \alpha)\right) \phi_{0}}\right. \\
& \left.\times e^{-\kappa /(2 \alpha) \phi}\right\} .
\end{aligned}
$$

The mass function may be given a better parametrization in terms of its critical value such that

$$
\left.\frac{d M}{d \phi}\right|_{\phi=\phi_{c}}=0 .
$$

The critical value, $\phi_{C}$, may be determined from the relation

$$
e^{\left(\left(\kappa+6 \alpha^{2}\right) /(2 \alpha)\right) \phi_{0}}=\frac{12 \alpha^{2}}{6 \alpha^{2}-\kappa} e^{\left(\left(\kappa+6 \alpha^{2}\right) /(2 \alpha)\right) \phi_{C}} .
$$

Therefore we may write 


$$
\begin{aligned}
n M(\phi)= & \frac{2 \kappa V_{0} R_{0}{ }^{3}}{\kappa+6 \alpha^{2}} e^{3 \alpha \phi_{C}}\left\{e^{3 \alpha\left(\phi-\phi_{C}\right)}\right. \\
& \left.+\frac{6 \alpha^{2}}{\kappa} e^{-(\kappa / 2 \alpha)\left(\phi-\phi_{C}\right)}\right\} .
\end{aligned}
$$

The minimum mass value is

$$
n M\left(\phi_{C}\right)=2 V_{0} R_{0}^{3} e^{3 \alpha \phi_{C}} .
$$

Differentiating with respect to $\phi$ we find

$$
n \frac{d M}{d \phi} e^{-3 \alpha \phi}=\frac{6 \alpha \kappa V_{0} R_{0}{ }^{3}}{\kappa+6 \alpha^{2}}\left\{1-e^{-\left(\left(\kappa+6 \alpha^{2}\right) / 2 \alpha\right)\left(\phi-\phi_{C}\right)}\right\} .
$$

It is now possible to substitute this expression into (17) and verify that it is identically satisfied. This is a consistency check.

To complete the solution, we go to the Dirac system:

$$
\begin{aligned}
& \dot{h}_{1}+\frac{3 \dot{R}}{2 R} h_{1}+M(\phi) h_{2}=0, \\
& \dot{h}_{2}+\frac{3 \dot{R}}{2 R} h_{2}-M(\phi) h_{1}=0 .
\end{aligned}
$$

Defining $X(t)=e^{(3 / 2) \alpha \phi} h_{1}(t)$ and $Y(t)=e^{(3 / 2) \alpha \phi} h_{2}(t)$ the above system can be written in matrix form:

$$
\frac{d}{d t}\left(\begin{array}{c}
X(t) \\
Y(t)
\end{array}\right)=\left(\begin{array}{cc}
0 & -M(\phi) \\
M(\phi) & 0
\end{array}\right)\left(\begin{array}{l}
X(t) \\
Y(t)
\end{array}\right) .
$$

This can be integrated formally and the general solution reads

$$
\begin{aligned}
& h_{1}(t)=e^{-(3 / 2) \alpha \phi}\{A \cos \Omega(t)+B \sin \Omega(t)\} \\
& h_{2}(t)=e^{-(3 / 2) \alpha \phi}\{A \sin \Omega(t)-B \cos \Omega(t)\}
\end{aligned}
$$

where $A=X(0), B=Y(0)$ are the initial values and

$$
\Omega(t)=\int_{0}^{t} M\left(t^{\prime}\right) d t^{\prime}
$$

The mass is a complicated function of $t$, so we will leave the solution implicit in terms of $\Omega(t)$. In fact the Majorana spinor field for the solution is of the form

$$
\psi=e^{-(3 / 2) \alpha \phi+\gamma_{0} \Omega}\left(A-B \gamma_{o}\right) \xi .
$$

To conclude, we work out the expansion function as a function of $t$

$$
\begin{aligned}
R(t)= & R_{0} e^{\alpha \phi_{C}} \mid \frac{12 \alpha^{2}}{6 \alpha^{2}-\kappa} \\
& \times\left.\sin ^{2}\left(\sqrt{\frac{\kappa V_{0}}{8 \alpha^{2}}\left(\kappa+6 \alpha^{2}\right)} t\right)\right|^{\left(2 \alpha^{2}\right) /\left(\kappa+6 \alpha^{2}\right)} .
\end{aligned}
$$

It is not difficult to determine the following observable quantities; the Hubble parameter:

$$
H(t) \equiv \frac{\dot{R}}{R}=\sqrt{\frac{2 \kappa \alpha^{2} V_{0}}{\kappa+6 \alpha^{2}}} \cot \left(\sqrt{\frac{\kappa V_{0}}{8 \alpha^{2}}\left(\kappa+6 \alpha^{2}\right)}\right),
$$

and the acceleration parameter:

$$
\frac{R \ddot{R}}{\dot{R}^{2}}=1-\frac{\kappa+6 \alpha^{2}}{4 \alpha^{2}} \csc ^{2}\left(\sqrt{\frac{\kappa V_{0}}{8 \alpha^{2}}\left(\kappa+6 \alpha^{2}\right)} t\right) .
$$

In the limit where $\kappa \ll \alpha^{2}$ and $V_{0} \sim 1$, to the first order of approximation we would have

$$
R(t) \sim t^{2 / 3},
$$

i.e. a matter-dominated Friedmann universe.

We next consider the case of a cosmological constant: $V_{0}=-\Lambda$. In this case Eqs. (15)-(17) can be considered representing a generalization of the $\Lambda_{\mathrm{CDM}}$ model [9]. The solution of Eq. (15) now becomes

$$
\dot{\phi}(t)=\sqrt{\frac{2 \kappa \Lambda}{\kappa+6 \alpha^{2}}} \operatorname{coth}\left(\sqrt{\frac{\kappa \Lambda}{8 \alpha^{2}}\left(\kappa+6 \alpha^{2}\right)}\right),
$$

yielding

$$
\phi(t)=\phi_{0}+\frac{2 \alpha}{\kappa+6 \alpha^{2}} \ln \left|\sinh ^{2}\left(\sqrt{\frac{\kappa \Lambda}{8 \alpha^{2}}\left(\kappa+6 \alpha^{2}\right) t}\right)\right|,
$$

and

$$
\operatorname{coth}^{2}\left(\sqrt{\frac{\kappa \Lambda}{8 \alpha^{2}}\left(\kappa+6 \alpha^{2}\right) t}\right)=e^{-\left(\left(\kappa+6 \alpha^{2}\right) / 2 \alpha\right)\left(\phi-\phi_{0}\right)}+1 .
$$

In this case we find the mass function to be

$$
\begin{aligned}
n M(\phi)= & \frac{2 \kappa \Lambda R_{0}^{3}}{\kappa+6 \alpha^{2}}\left\{-e^{3 \alpha \phi}+\frac{6 \alpha^{2}-\kappa}{2 \kappa} e^{\left(\left(\kappa+6 \alpha^{2}\right) / 2 \alpha\right) \phi_{0}}\right. \\
& \left.\times e^{-(\kappa / 2 \alpha) \phi}\right\} .
\end{aligned}
$$

The rest of the solutions of the Dirac system are unchanged. The expansion function now has the form

$$
R(t)=R_{0} e^{\alpha \phi_{0}}\left[\sinh \left(\sqrt{\frac{\kappa \Lambda}{8 \alpha^{2}}\left(\kappa+6 \alpha^{2}\right)}\right)\right]^{4 \alpha^{2} /\left(\kappa+6 \alpha^{2}\right)} .
$$

We also note the forms of the Hubble parameter

$$
H(t)=\sqrt{\frac{2 \kappa \alpha^{2} \Lambda}{\kappa+6 \alpha^{2}}} \operatorname{coth}\left(\sqrt{\frac{\kappa \Lambda}{8 \alpha^{2}}\left(\kappa+6 \alpha^{2}\right)} t\right),
$$

and the acceleration parameter:

$$
\frac{R \ddot{R}}{\dot{R}^{2}}=1-\frac{\kappa+6 \alpha^{2}}{4 \alpha^{2}} \cosh ^{-2}\left(\sqrt{\frac{\kappa \Lambda}{8 \alpha^{2}}\left(\kappa+6 \alpha^{2}\right) t}\right) .
$$

In the limit $\kappa \ll \alpha^{2}$ and $\Lambda \sim 1$, Eq. (39) again yields the 
matter-dominated Friedmann universe, $R(t) \sim t^{2 / 3}$. In this limit (for $t<t_{\Lambda}$ ) we get the Hubble parameter to be

$$
H(t) \sim \frac{1}{t_{\Lambda}} \operatorname{coth}\left(t / t_{\Lambda}\right),
$$

and the expansion factor to be

$$
R(t) \sim \sinh ^{2 / 3}\left(\frac{t}{t_{\Lambda}}\right)
$$

where $t_{\Lambda}^{-1}=(\sqrt{3 \kappa} \bar{\Lambda}) / 2$. These values of the Hubble parameter and the expansion factor are those obtained in the flat $\Lambda_{\mathrm{CDM}}$ model [9]. Hence the present model can be considered as a generalization of the $\Lambda_{\mathrm{CDM}}$ model.

\section{CONCLUSIONS}

In this paper we found exact solutions to a cosmological model where a neutral scalar field and a Majorana fermion field are coupled to gravity represented by the RobertsonWalker metric. Our study was motivated by the massvarying neutrino models where the scalar field could rep- resent the acceleron field and the Majorana fermion field the neutrino. However, our results are valid in a broader context, generalizing the earlier solution of Isham and Nelson. The exact solution we found has a number of parameters: the constants $R_{0}$ and $\alpha$ of the expansion function and the critical value of the scalar field, $\phi_{c}$. These parameters can be adjusted to construct different phenomenological models.

\section{ACKNOWLEDGMENTS}

This work was supported in part by the U.S. National Science Foundation Grants No. INT-0352192 and PHY0555231 at the University of Wisconsin, and in part by the University of Wisconsin Research Committee with funds granted by the Wisconsin Alumni Research Foundation. We also acknowledge support through TUBITAK-NSF Joint Research Project TBAG-U/84(103T113). We thank $\varnothing$. Grøn for suggesting the solution with $V_{0}=-\Lambda$ and for bringing Ref. [9] to our attention.
[1] D. B. Kaplan, A.E. Nelson, and N. Weiner, Phys. Rev. Lett. 93, 091801 (2004).

[2] R. Fardon, A.E. Nelson, and N. Weiner, J. Cosmol. Astropart. Phys. 10 (2004) 005.

[3] G. R. Farrar and P. J.E. Peebles, Astrophys. J. 604, 1 (2004).

[4] R. D. Peccei, Phys. Rev. D 71, 023527 (2005).
[5] N. Afshordi, M. Zaldarriaga, and K. Kohri, Phys. Rev. D 72, 065024 (2005).

[6] R. Horvat, J. Cosmol. Astropart. Phys. 01 (2006) 015.

[7] A.W. Brookfield, C. van de Bruck, D.F. Mota, and D. Tocchini-Valentini, Phys. Rev. D 73, 083515 (2006).

[8] C. J. Isham and J. E. Nelson, Phys. Rev. D 10, 3226 (1974).

[9] Ø. Grøn, Eur. J. Phys. 23, 135 (2002). 